\title{
The Heat of Transfer in a Chemical Reaction at Equilibrium
}

\author{
Jing Xu, Signe Kjelstrup*, Dick Bedeaux, and Jean-Marc Simon ${ }^{a}$ \\ Department of Chemistry, Faculty of Natural Science and Technology, \\ Norwegian University of Science and Technology, N-7491 Trondheim, Norway \\ a On leave from the Laboratoire de Recherches sur la Reactivite des Solides, \\ UMR 5613 CNRS University of Bourgogne, BP 47870, F-21078 Dijon Cedex, \\ France
}

*Corresponding author (signe.kjelstrup@chem.ntnu.no)

\begin{abstract}
We study a reacting mixture $\left(2 \mathrm{~F} \leftrightarrow \mathrm{F}_{2}\right)$ in a temperature gradient. We had previously used boundary-driven non-equilibrium molecular dynamics (NEMD) simulations to study this system, and found that the reaction was close to local chemical equilibrium in temperature gradients up to $10^{12} \mathrm{~K} / \mathrm{m}$. Using the condition of local chemical equilibrium, we show that the heat of transfer of the reacting mixture is equal to minus the enthalpy of the reaction. The fact that the sign of the heat of transfer is determined by the type of reaction adds insight to the discussion of the origin of the sign.
\end{abstract}

\section{Introduction}

The Soret effect is defined in classical non-equilibrium thermodynamics as a mass flux due to a temperature gradient. The heat of transfer, the thermal diffusion factor, the thermal diffusion coefficient, or the Soret coefficient have all been used to quantify this effect [1-6]. The sign of the Soret coefficient determines where the transported component will accumulate in the temperature gradient; on the hot or on the cold side. Much effort has been devoted toward finding models that explain this sign. The original model of Furry et al. [4], developed for isotope separation purposes, explained the separation as a pure mass effect. The successful model of Kempers, developed for hydrocarbon separation [5], contains thermodynamic variables only. In their work on non-ideal mixtures, Rousseau et al. [6] found that the enthalpy of mixing was

J. Non-Equilib. Thermodyn. $\cdot 2007 \cdot$ Vol. $32 \cdot$ No. 3

(C) Copyright 2007 Walter de Gruyter $\cdot$ Berlin $\cdot$ New York. DOI 10.1515/JNETDY.2007. II 
central for the sign. Against this background, one may ask whether the effect is kinetic or thermodynamic or both.

Non-equilibrium molecular dynamics (NEMD) simulations offer a good opportunity to study molecular mechanisms for separation in temperature gradients [1]. In an earlier paper [7], we performed NEMD simulations of a reacting mixture in a temperature gradient using the 2- and 3-body potential of Stillinger and Weber [8]. The reaction

$$
2 \mathrm{~F} \leftrightarrow \mathrm{F}_{2},
$$

which is strongly exothermic, was modelled without quantum mechanical effects, using interaction potentials that reproduced the essence of a chemical reaction: the two-way traffic across an activation energy barrier. We found that the reaction was close to chemical equilibrium everywhere in large temperature gradients, typically $10^{11} \mathrm{~K} / \mathrm{m}$ [7]. De Groot and Mazur [2] gave transport equations for a chemical reaction in a temperature gradient, and showed that the enthalpy of reaction gives a contribution to the Soret effect but does not explain all of the separation. The model system, developed by us, shall be used here to add numerical insight to a limiting case of their system, namely the case where the reaction is in chemical equilibrium.

In the NEMD simulations, we applied different temperature gradients (between $10^{11}$ and $10^{12} \mathrm{~K} / \mathrm{m}$ ) to a supercritical reacting fluid, with an overall particle density near $11 \mathrm{kmol} / \mathrm{m}^{3}$. Examples of temperature profiles calculated from the system's local kinetic temperature are shown in Figure 1.

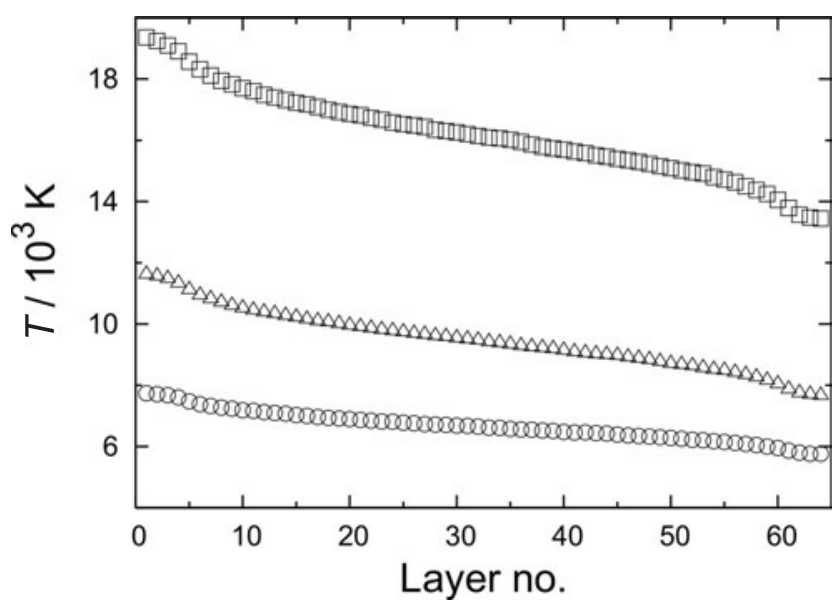

Figure 1 Temperature profiles in the reacting system. The overall density is $C_{\mathrm{F}}+2 C_{\mathrm{F} 2}=$ $11271 \mathrm{~mol} / \mathrm{m}^{3}$. The temperature gradients are $2 \times 10^{11} \mathrm{~K} / \mathrm{m}$ (circles), $4 \times 10^{11} \mathrm{~K} / \mathrm{m}$ (triangles), and $6 \times 10^{11} \mathrm{~K} / \mathrm{m}$ (squares). 


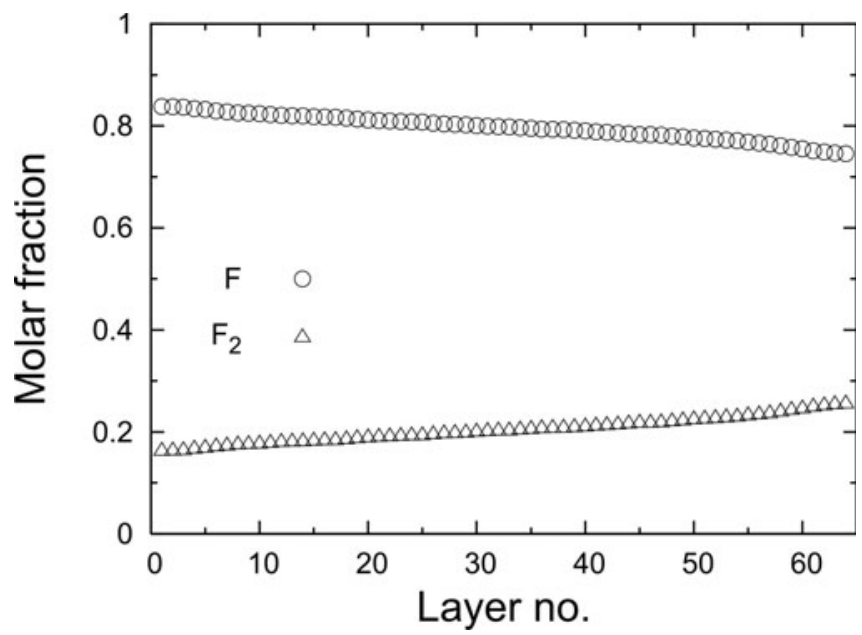

Figure 2 Molar fractions of component $\mathrm{F}$ and $\mathrm{F}_{2}$ in the reacting system at overall density $C_{\mathrm{F}}+2 C_{\mathrm{F} 2}=11271 \mathrm{~mol} / \mathrm{m}^{3}$. The temperature gradient is $2 \times 10^{11} \mathrm{~K} / \mathrm{m}$.

Even under the extreme conditions we used, we found that the chemical reaction was near local chemical equilibrium, especially in the centre of the box [7], with $\Delta_{\mathrm{r}} G \ll R T$.

The stationary state of the system was interesting. We observed an accumulation of the heavy species on the cold side and the light species on the hot side (see Figure 2), apparently in agreement with the mass effect predicted by Furry et al. [4]. The net mass flux was zero. But, in order to compensate for the effect of the chemical reaction, a net velocity of both reacting species was present, giving $J_{\mathrm{F}}=-2 J_{\mathrm{F} 2}$. An example of the component fluxes is shown in Figure 3. The molar flux of a fluorine molecule (the heavy particle) was directed to the hot side, and the molar flux of a fluorine atom (the light particle) was directed to the cold side. These flux directions are the opposite of those predicted by the mass effect, but in agreement with the gradient that is established. We shall see that the distribution in the temperature gradient can be understood as a stationary-state Soret effect, with its origin in the properties of the chemical reaction, and that the enthalpy of the reaction determines its sign.

\section{Theory}

The system has transports of heat and mass (with $J_{\mathrm{F}}=-2 J_{\mathrm{F} 2}$ ) and a chemical reaction with two components in equilibrium. There are no externally applied forces and the transports are one-dimensional. The entropy production is therefore [2] 


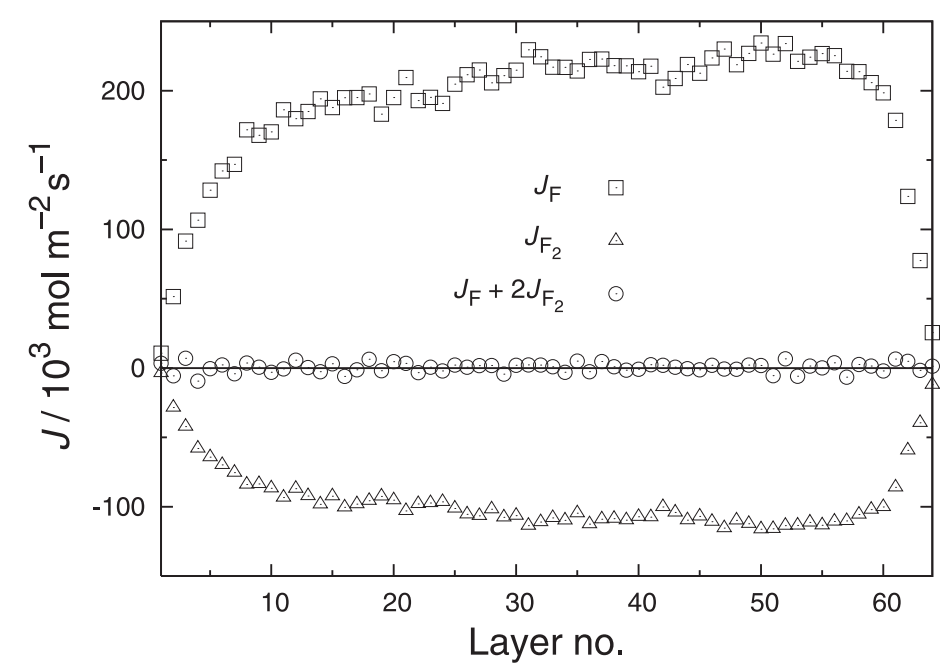

Figure 3 The molar fluxes in the reacting system simulated by NEMD with a temperature gradient of $2 \times 10^{11} \mathrm{~K} / \mathrm{m}$ (see Figure 1). The squares give the positive molar flux of $\mathrm{F}$ and the triangles give the negative molar flux of $F_{2}$. The circles give the total molar flux or the mass flux, which is zero.

$$
\sigma=J_{q}^{\prime} \nabla\left(\frac{1}{T}\right)-\frac{1}{T} J_{\mathrm{F}_{2}}\left(\nabla \mu_{\mathrm{F}_{2}, T}-2 \nabla \mu_{\mathrm{F}, T}\right)
$$

Here $J_{q}^{\prime}$ is the measurable heat flux, $T$ the absolute temperature, $J_{k}$ the component flux of $\mathrm{F}$ or $\mathrm{F}_{2}$, and $\nabla \mu_{k, T}$ is the gradient in chemical potential at constant temperature. De Groot and Mazur described a similar system in their monograph [2]. We haven chosen to introduce the simplification of chemical equilibrium in the outset, and have a simpler form of the entropy production than they have. With the condition $\Delta_{\mathrm{r}} G \equiv \mu_{\mathrm{F} 2}-2 \mu_{\mathrm{F}}=H_{\mathrm{F} 2}-2 H_{\mathrm{F}}-$ $T\left(S_{\mathrm{F} 2}-2 S_{\mathrm{F}}\right)=0$, the chemical driving force can be further written as

$$
\nabla\left(\mu_{\mathrm{F}_{2}}-2 \mu_{\mathrm{F}}\right)_{T}=\left(S_{\mathrm{F}_{2}}-2 S_{\mathrm{F}}\right) \nabla T=\frac{1}{T}\left(H_{\mathrm{F}_{2}}-2 H_{\mathrm{F}}\right) \nabla T
$$

By introducing this, the entropy production becomes a function of one flux and one force only:

$$
\sigma_{\Delta_{r} G=0}=J_{q}^{\prime} \nabla\left(\frac{1}{T}\right)-\frac{1}{T} J_{\mathrm{F}_{2}}\left(S_{\mathrm{F}_{2}}-2 S_{\mathrm{F}}\right) \nabla T=J_{q} \nabla\left(\frac{1}{T}\right)
$$

The entropy production becomes zero when the temperature gradient vanishes. This is because the fluxes of the fluorine atom and molecule are driven 
The Heat of Transfer in a Chemical Reaction at Equilibrium

solely by the temperature gradient. The non-isothermal reacting mixture has, in its stationary state, a varying flux of each component across the box, but the mass flux is always zero; see Figure 3. The total heat flux is

$$
J_{q}=J_{q}^{\prime}+J_{\mathrm{F}_{2}}\left(H_{\mathrm{F}_{2}}-2 H_{\mathrm{F}}\right)=J_{q}^{\prime}+J_{\mathrm{F}_{2}}\left(S_{\mathrm{F}_{2}}-2 S_{\mathrm{F}}\right) T .
$$

From Eq. (2) we obtain the linear laws

$$
\begin{aligned}
& \nabla\left(\frac{1}{T}\right)=r_{q q} J_{q}^{\prime}+r_{q \mu} J_{\mathrm{F}_{2}} \\
& -\frac{1}{T}\left(\nabla \mu_{\mathrm{F}_{2}, T}-2 \nabla \mu_{\mathrm{F}, T}\right)=r_{\mu q} J_{q}^{\prime}+r_{\mu \mu} J_{\mathrm{F}_{2}}
\end{aligned},
$$

where $r_{i j}$ are the resistivities subject to Onsager's reciprocal relations. Alternatively, from Eq. (4) we obtain

$$
\nabla\left(\frac{1}{T}\right)=R_{q q} J_{q}
$$

The coefficients in Eq. (6) must be related because the fluxes are dependent. Using the relation (5) we obtain

$$
\nabla\left(\frac{1}{T}\right)=R_{q q} J_{q}^{\prime}+R_{q q}\left(H_{\mathrm{F}_{2}}-2 H_{\mathrm{F}}\right) J_{\mathrm{F}_{2}}
$$

It follows that

$$
r_{q q}=R_{q q} \quad \text { and } \quad r_{q \mu}=R_{q q}\left(H_{\mathrm{F}_{2}}-2 H_{\mathrm{F}}\right)
$$

Using Eqs. (3) and (6a), we furthermore have

$$
\begin{gathered}
-\frac{1}{T}\left(\nabla \mu_{\mathrm{F}_{2}, T}-2 \nabla \mu_{\mathrm{F}, T}\right) \\
=\left(H_{\mathrm{F}_{2}}-2 H_{\mathrm{F}}\right) \nabla\left(\frac{1}{T}\right)=R_{q q}\left(H_{\mathrm{F}_{2}}-2 H_{\mathrm{F}}\right) J_{q} \\
=R_{q q}\left(H_{\mathrm{F}_{2}}-2 H_{\mathrm{F}}\right) J_{q}^{\prime}+R_{q q}\left(H_{\mathrm{F}_{2}}-2 H_{\mathrm{F}}\right)^{2} J_{\mathrm{F}_{2}} .
\end{gathered}
$$

It follows that

J. Non-Equilib. Thermodyn. $\cdot 2007 \cdot$ Vol. $32 \cdot$ No. 3 


$$
r_{\mu q}=R_{q q}\left(H_{\mathrm{F}_{2}}-2 H_{\mathrm{F}}\right)=r_{q \mu} \quad \text { and } \quad r_{\mu \mu}=R_{q q}\left(H_{\mathrm{F}_{2}}-2 H_{\mathrm{F}}\right)^{2} .
$$

We can now define the heat of transfer from Eq. (6a) and identify its value using Eqs. (9) and (11):

$$
q^{*}=\left[\frac{J_{q}^{\prime}}{J_{\mathrm{F}_{2}}}\right]_{\nabla T=0}=-\frac{r_{q \mu}}{r_{q q}}=-\left(H_{\mathrm{F}_{2}}-2 H_{\mathrm{F}}\right) .
$$

The heat of transfer is simply equal to minus the reaction enthalpy, a quantity that varies with temperature and composition. The distribution of the components in the temperature gradient is thus decided by the nature of the reaction. An exothermic reaction has a positive heat of transfer, and an endothermic reaction has a negative heat of transfer, when the reaction is in local chemical equilibrium everywhere in the system. One cannot speak of a Soret equilibrium in the traditional sense in the system, i.e., a balance of chemical and thermal forces at zero mass flux. This is because the component fluxes are non-zero (see Figure 2). They are still time-independent. One must rather speak of a Soret stationary state. We can estimate the heat of transfer from the reaction enthalpy change in Eq. (12). In the present case, the ratio of fluxes is a ratio of zero over zero in the limit of zero temperature gradient. This ratio is finite, however, as shown.

\section{Calculations}

The reaction model and the NEMD method have already been described in detail [7-9]. The system had initially 1000 reacting fluorine atoms in a noncubic (MD) box divided into 128 equal planar layers, symmetrical around the centre of the box. The overall density was $11271 \mathrm{~mol} / \mathrm{m}^{3}$. NEMD simulations were performed with four different temperature gradients, see Table 1 and ref. [7]. The enthalpy change of the reaction was estimated from the molar enthalpies of component $\mathrm{F}$ and $\mathrm{F}_{2}$ computed by Eq. (13):

$$
H_{l}=N_{A}\left\{\frac{1}{N_{l}} \sum_{i \in l} \Phi_{i}+\frac{p_{l} V_{L}}{N_{l}}+\frac{3}{2} k_{B} T\right\} .
$$

Table 1 Temperature gradients and corresponding total heat flux and pressure.

\begin{tabular}{ccllc}
\hline$T_{H} / 10^{3} \mathrm{~K}$ & $T_{L} / 10^{3} \mathrm{~K}$ & $\nabla T / 10^{11} \mathrm{~K} \mathrm{~m}^{-1}$ & $J_{q} / 10^{8} \mathrm{~kJ} \mathrm{~m}^{-2} \mathrm{~s}^{-1}$ & $P / 10^{3} \mathrm{bar}$ \\
\hline 5.8 & 4.8 & 1 & 0.65 & 5.03 \\
7.7 & 5.8 & 2 & 1.60 & 6.33 \\
11.6 & 7.7 & 4 & 4.03 & 8.93 \\
19.3 & 13.5 & 6 & 8.49 & 15.2 \\
\hline
\end{tabular}




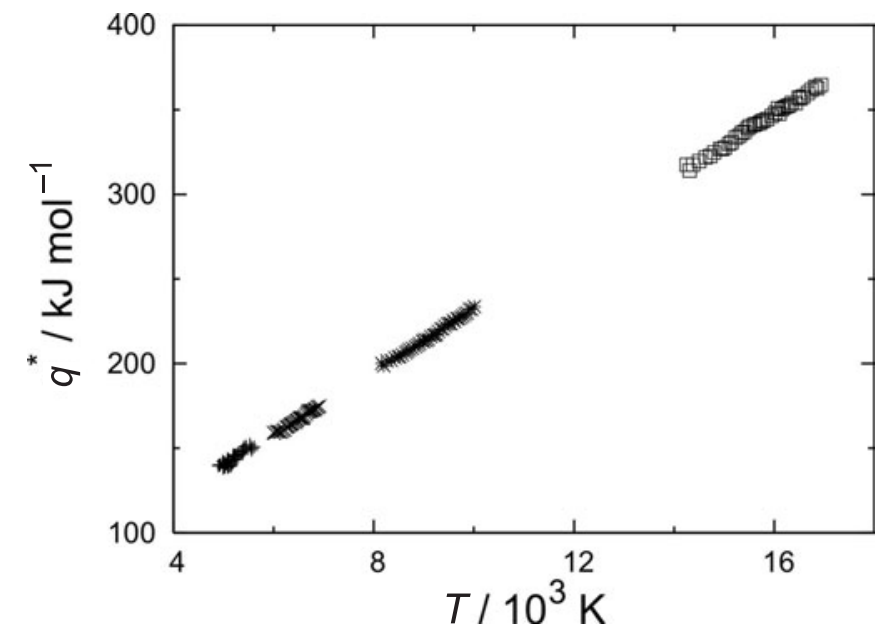

Figure 4 The heat of transfer for the exothermic reacting mixture in a closed box, for the four temperature gradients that were investigated (see Table 1).

The heats of transfer for the four series of measurements were given by Eq. (12). The results of the calculations gave a positive heat of transfer (the reaction is exothermic); see Figure 4.

\section{Discussion and conclusions}

Figure 3 shows that the presence of a simple reaction between two components leads to non-zero, but time-independent fluxes of reactants and product in a stationary temperature gradient. We have shown how we can define the Soret effect for the stationary state. The distribution of components in space, and the values of their fluxes, must all be explained by the particular system and its constraints. The distribution shown in Figure 2 has more of the heavy component on the cold side and more of the light component on the hot side. It is tempting in this situation to explain the distribution by a mass effect, see, e.g., [3]. On the basis of Eq. (12), we can say, however, that the dominant reason for the distribution in this particular case is related to enthalpy effects, not mass effects.

With equilibrium in the chemical reaction, the entropy production of the system becomes a function of only one flux and one force. The thermal force is the driving force for all fluxes. The condition $\Delta_{r} G=0$ gave a heat of transfer equal to minus the reaction enthalpy. We therefore know the sign of the heat of transfer for a chemical reaction at local equilibrium in a temperature gradient. The distribution of components in the temperature gradient is determined by this sign, when the chemical reaction is in equilibrium. It may be fruitful to see the sign and value as a response to the boundary conditions.

J. Non-Equilib. Thermodyn. $\cdot 2007 \cdot$ Vol. $32 \cdot$ No. 3 
That the components carry latent heat across the box is a response to the boundary conditions. By their diffusion in the temperature gradient (thermal diffusion), they contribute to a more efficient conductivity of heat. The nonzero, time-independent component fluxes can be understood, as already explained by de Groot and Mazur [2], as ways to carry extra cooling capacity to the hot side, and extra heating capacity to the cold side.

Studies of isotope mixtures (without a chemical reaction) [see refs. 1, 9 and references therein] revealed that the contribution from the lighter component to the heat flux of Eq. (7) is predominantly kinetic energy, and this contribution increases from the cold to the hot side. The contribution from the heavier component to the heat flux is predominantly energy transfer by molecular collisions and it increases from the hot to the cold side. Accordingly, the Soret effect was interpreted as the system's response to external thermal force, by minimising its entropy production at stationary state; heat was conducted more effectively through the system with the lighter component on the hot side. This line of thinking can be used here also. Heat is conducted more effectively by allowing non-zero mass fluxes that carry enthalpy across the box.

The NEMD simulations were used here to estimate the heat of transfer for the reacting mixture in chemical equilibrium. The results show a linear variation of the heat of transfer with temperature. The four runs refer to different pressures, but this does not seem to have an effect. We have no good explanation of this at the moment.

With the simple example that we have taken, the mode of heat conduction is special. In this case, when the reaction is not in equilibrium, other variables may add to the explanation of the transport and the resulting profiles. The effect of a non-zero reaction Gibbs energy shall be discussed in the future.

\section{Acknowledgement}

The Research Council of Norway is thanked for grant no. 167336/V30 "Transport at a nano-scale at surfaces and contact lines".

\section{References}

[1] Hafskjold, B., Computer simulations of thermal diffusion in binary fluid mixtures, Chap. 1, in: Concepts, Theory, and Computer Simulations (Lecture Notes in Physics), Eds. W. Köhler, S. Wiegand, pp. I-!, Springer, Berlin, 2002.

[2] de Groot, S.R., Mazur, P., Non-Equlibrium Thermodynamics, I, Dover, 1984.

[3] Førland, K.S., Førland, T., Kjelstrup, S., Irreversible Thermodynammics, Theory and Application, I, Tapir, 2001. 
The Heat of Transfer in a Chemical Reaction at Equilibrium

[4] Furry, W.H., Jones, R.C., Onsager, L., On the theory of isotope separation by thermal diffusion, Phys. Rev., 55 (1939), 1083-1095.

[5] Kempers, L.J.T.M., A comprehensive theory of the Soret effect in a multicomponent mixture, Chap. 5, in: Concepts, Theory, and Computer Simulations (Lecture Notes in Physics), Eds. W. Kõhler, S. Wiegand, pp. I-I, Springer, Berlin, 2002.

[6] Rousseau, B., Niete-Draghi, C., Bonet Avalos, J., The role of molecular association in the change of sign of the Soret coefficient in aqueous mixtures, Europhysics Lett., 67 (2004), 976-982.

[7] Xu, J., Kjelstrup, S., Bedeaux, D., Molecular dynamics simulations of a chemical reaction; conditions for local equilibrium in a temperature gradient, Phys. Chem. Chem. Phys., 8 (2006), 2017-2027.

[8] Stillinger, F.H., Weber, T.A., Molecular dynamics simulation for chemically reactive substances. Fluorine, J. Chem. Phys., 88 (1988), 5123-5133.

[9] Hafskjold, B., Ikeshoji, T., Ratkje, S.K., On the molecular mechanism of thermal-diffusion in liquids, Mol. Phys., 80 (1993), 1389-1412.

Paper received: 2006-09-17

Paper accepted: 2006-12-13 


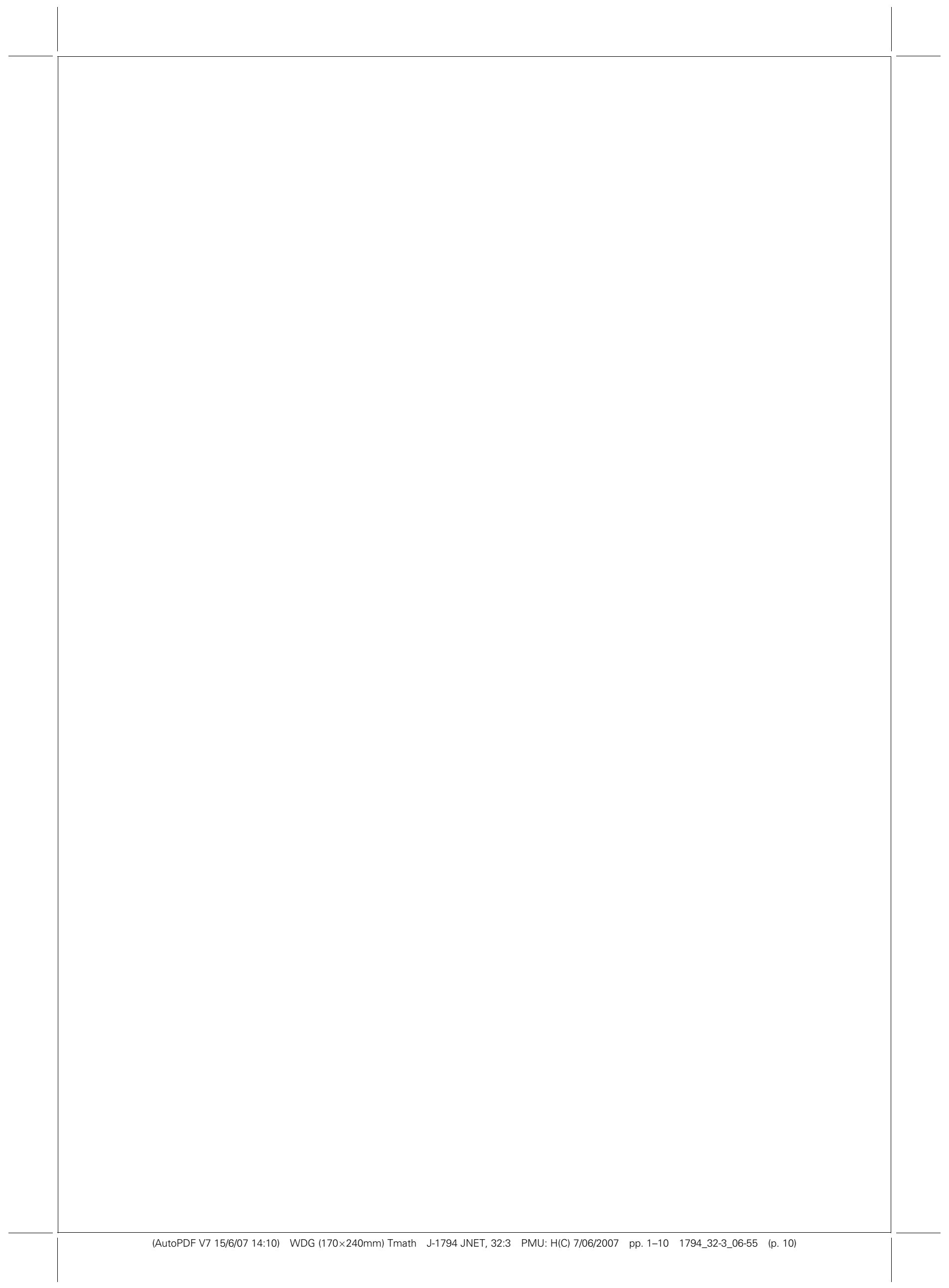

